\title{
Damped wave equation and dissipative wave equation in fractal strings within the local fractional variational iteration method
}

\author{
Wei-Hua Su ${ }^{1,2}$, Dumitru Baleanu ${ }^{3,4,5}$, Xiao-Jun Yang ${ }^{6,7,8^{*}}$ and Hossein Jafari ${ }^{9}$
}

\section{"Correspondence:}

dyangxiaojun@163.com

${ }^{6}$ Department of Mathematics and

Mechanics, China University of

Mining and Technology, Xuzhou, Jiangsu 221008, China

${ }^{7}$ Institute of Software Science, Zhengzhou Normal University,

Zhengzhou, 450044, China

Full list of author information is

available at the end of the article

\begin{abstract}
In this paper, the local fractional variational iteration method is given to handle the damped wave equation and dissipative wave equation in fractal strings. The approximation solutions show that the methodology of local fractional variational iteration method is an efficient and simple tool for solving mathematical problems arising in fractal wave motions.
\end{abstract}

MSC: $74 \mathrm{H} 10 ; 35 \mathrm{~L} 05 ; 28 \mathrm{~A} 80$

Keywords: local fractional variational iteration method; damped wave equation; dissipative wave equation; local fractional operators; fractal strings

\section{Introduction}

The variational iteration method was effectively applied in various fields of science and engineering [1-15] and the references therein. It is in some cases, more powerful than the existing techniques, e.g., the fractional variational iteration method $[6,16,17]$, the homotopy perturbation method $[18,19]$, the exp-function method [20, 21], the decomposition method [22-24], the homotopy analysis method [25, 26] and others [27]. The wave equation was investigated within some differential methods $[7-15,18-26]$ and the references therein.

As it is known, the quantum behavior of microphysics in terms of a non-differentiable space-time continuum possesses and has fractal property. Also, it was shown by many authors that a time-space structure of microphysics is non-differentiable. The relativistic quantum mechanics in fractal time space was suggested in [28]. It was pointed out that, while the zero set represents the Cantor point-like quantum particle, the empty set was the basic mathematical representation of the quantum wave [29]. The exact solutions for a class of fractal time random walks were researched in [30]. The questions of a philosophical nature about fractal spacetime and its implications for phenomenology and ontology were shown in [31]. The fractal time-space structure for dealing with the non-differentiability and infinities of fractals derived from local fractional operators was presented in [32-34] and the references therein. A solution of the wave equation in fractal vibrating string by using the local fractional Fourier series was discussed in [35]. The diffusion equation on Cantor time-space was reported in [36] while the diffusion problems on fractal space were suggested in [37]. The heat conduction problem by local fractional variational iteration method was investigated in [38]. The heat conduction equation in

(c) 2013 Su et al.; licensee Springer. This is an Open Access article distributed under the terms of the Creative Commons Attribution License (http://creativecommons.org/licenses/by/2.0), which permits unrestricted use, distribution, and reproduction in any medium, provided the original work is properly cited. 
fractal time space was structured in [32]. A relaxation equation in fractal space was set up in [39]. The anomalous diffusion equation in the fractal time-space fabric was pointed out in [40]. The Fokker-Planck equation in fractal time was considered in [41].

Recently, fractional calculus analysis and fractional dynamics are hot topics [42-48]. In this paper, we consider a general wave equation of a fractal string within the local fractional operators, namely

$$
L_{\zeta \zeta}^{(2 \alpha)} u(\zeta, \xi)+R_{\xi}^{(\alpha)} u(\zeta, \xi)-g(\zeta, \xi)=0
$$

where [32-35]

$$
\frac{\partial^{2 \alpha}}{\partial \xi^{2 \alpha}} u(\zeta, \xi)=\overbrace{\frac{\partial^{\alpha}}{\partial \xi^{\alpha}} \frac{\partial^{\alpha}}{\partial \xi^{\alpha}}}^{2 \text { times }} u(\zeta, \xi),
$$

and where $R_{\xi}^{(\alpha)}$ is a local fractional linear operator, which has low order local fractional partial derivatives with respect to $\xi$, subject to fractal initial conditions

$$
\frac{\partial^{i \alpha}}{\partial \zeta^{i \alpha}} u(\zeta, \xi)=\varphi_{i}(\zeta), \quad i \in N_{0}
$$

Thus, we obtain

$$
\begin{aligned}
& L_{\xi \xi}^{(2 \alpha)} u(\zeta, \xi)=L_{t t}^{(2 \alpha)} u(x, t), \quad R_{\xi}^{(\alpha)} u(\zeta, \xi)=-L_{t}^{(\alpha)} u(x, t), \\
& g(\zeta, \xi)=L_{x x}^{(2 \alpha)} u(x, t)+L_{x}^{(\alpha)} u(x, t)+m(x, t),
\end{aligned}
$$

and we have the following dissipative wave equation in fractal time space:

$$
L_{t t}^{(2 \alpha)} u(x, t)-L_{t}^{(\alpha)} u(x, t)-L_{x x}^{(2 \alpha)} u(x, t)-L_{x}^{(\alpha)} u(x, t)-m(x, t)=0, \quad 0 \leq x \leq l, t>0,
$$

subject to initial conditions

$$
u(x, 0)=\mu_{1}(x), \quad L_{t}^{(\alpha)} u(x, 0)=\mu_{2}(x), \quad 0 \leq x \leq l .
$$

If we start with

$$
\begin{aligned}
& L_{\xi \xi}^{(2 \alpha)} u(\zeta, \xi)=L_{t t}^{(2 \alpha)} u(x, t), \quad R_{\xi}^{(\alpha)} u(\zeta, \xi)=-L_{t}^{(\alpha)} u(x, t), \\
& g(\zeta, \xi)=L_{x x}^{(2 \alpha)} u(x, t)+n(x, t),
\end{aligned}
$$

then we obtain the following damped wave equation given by

$$
L_{t t}^{(2 \alpha)} u(x, t)-L_{t}^{(\alpha)} u(x, t)-L_{x x}^{(2 \alpha)} u(x, t)-n(x, t)=0, \quad 0 \leq x \leq l, t>0,
$$

where the damping force is proportional to the velocity, $a$ and $b$ are constants, subject to initial conditions, which are suggested by the following expression:

$$
u(x, 0)=\psi_{1}(x), \quad L_{t}^{(\alpha)} u(x, 0)=\psi_{2}(x), \quad 0 \leq x \leq l .
$$


More recently, the local fractional variational iteration method, which was structured in [49], was applied to solve heat conduction equation on Cantor sets [38] and the local fractional Laplace equation [50]. The purpose of this paper is to present the solutions of the damped wave equation and the dissipative wave equation in fractal strings equipped with fractal initial conditions.

\section{Mathematical tools}

In this section, we recall briefly some basic theory of local fractional calculus, and for more details, see [32-36, 49-52].

Local fractional derivative of $f(x)$ at the point $x=x_{0}$, which is satisfied the condition $[32,35]$

$$
\left|f(x)-f\left(x_{0}\right)\right|<\varepsilon^{\alpha}
$$

with $\left|x-x_{0}\right|<\delta$, for $\varepsilon, \delta>0$ and $\varepsilon, \delta \in R$, is given by [32-36, 49-52]

$$
D_{x}^{(\alpha)} f\left(x_{0}\right)=f^{(\alpha)}\left(x_{0}\right)=\left.\frac{d^{\alpha} f(x)}{d x^{\alpha}}\right|_{x=x_{0}}=\lim _{x \rightarrow x_{0}} \frac{\Delta^{\alpha}\left(f(x)-f\left(x_{0}\right)\right)}{\left(x-x_{0}\right)^{\alpha}},
$$

where

$$
\Delta^{\alpha}\left(f(x)-f\left(x_{0}\right)\right) \cong \Gamma(1+\alpha) \Delta\left(f(x)-f\left(x_{0}\right)\right) .
$$

Now, Eq. (11) is written in the form [32]

$$
\Delta^{\alpha} f(x)=f^{(\alpha)}(x)(\Delta x)^{\alpha}+\lambda(\Delta x)^{\alpha}
$$

with $\lambda \rightarrow 0$ as $\Delta x \rightarrow 0$, or

$$
d^{\alpha} f=f^{(\alpha)}(x)(d x)^{\alpha} .
$$

Suppose that $f(x)$ is satisfied the condition (10) for $x \in[a, b]$, we can denote [32]

$$
f(x) \in C_{\alpha}(a, b) .
$$

The right-hand local fractional derivative is defined as [32-36, 49-52]

$$
x_{0}^{-} D_{x}^{\alpha} f(x)=f^{(\alpha)}\left(x_{0}^{-}\right)=\left.\frac{d^{\alpha} f(x)}{d x^{\alpha}}\right|_{x=x_{0}^{-}}=\lim _{x \rightarrow x_{0}^{-}} \frac{\Gamma(1+\alpha)\left[f(x)-f\left(x_{0}^{-}\right)\right]}{\left(x-x_{0}^{-}\right)^{\alpha}}
$$

if $f(x)$ is satisfied the conditions $x \in\left(x_{0}-\delta, x_{0}\right)$ and $f(x) \in C_{\alpha}[a, b]$.

The left-hand local fractional derivative is written as [32-36]

$$
x_{0}^{+} D_{x}^{\alpha} f(x)=f^{(\alpha)}\left(x_{0}^{+}\right)=\left.\frac{d^{\alpha} f(x)}{d x^{\alpha}}\right|_{x=x_{0}^{+}}=\lim _{x \rightarrow x_{0}^{+}} \frac{\Gamma(1+\alpha)\left[f(x)-f\left(x_{0}^{+}\right)\right]}{\left(x-x_{0}^{+}\right)^{\alpha}}
$$

if $f(x)$ is satisfied the conditions $f(x) \in C_{\alpha}[a, b]$ and $x \in\left(x_{0}, x_{0}+\delta\right)$. 
We can obtain that [32-36]

$$
\left.\frac{d^{\alpha}}{d x^{\alpha}} f(x)\right|_{x=x_{0}^{+}}=\left.\frac{d^{\alpha}}{d x^{\alpha}} f(x)\right|_{x=x_{0}^{-}}=\left.\frac{d^{\alpha}}{d x^{\alpha}} f(x)\right|_{x=x_{0}} .
$$

As an inverse local fractional derivative, local fractional integral of $f(x)$ at the point $x=x_{0}$ for $f(x) \in C_{\alpha}[a, b]$, is expressed by [32-36, 49-52]

$$
{ }_{a} I_{b}^{(\alpha)} f(x)=\frac{1}{\Gamma(1+\alpha)} \int_{a}^{b} f(t)(d t)^{\alpha}=\frac{1}{\Gamma(1+\alpha)} \lim _{\Delta t \rightarrow 0} \sum_{j=0}^{j=N-1} f\left(t_{j}\right)\left(\Delta t_{j}\right)^{\alpha},
$$

if there are conditions for a partition of the interval $[a, b]$ given by

$$
\Delta t_{j}=t_{j+1}-t_{j} \quad \text { and } \quad \Delta t=\max \left\{\Delta t_{1}, \Delta t_{2}, \Delta t_{j}, \ldots\right\} \quad \text { for } j=0, \ldots, N-1, t_{0}=a, t_{N}=b
$$

We always give the relation [32-36]

$$
f(x)={ }_{a} I_{x}^{(\alpha)} f^{(\alpha)}(x)
$$

with given conditions $f(x) \in C_{\alpha}[a, b]$ for $x \in(a, b)$.

Local fractional multiple integrals of $f(x)$ is given by [32-36]

$$
x_{0} I_{x}^{(k \alpha)} f(x)=\overbrace{x_{0} I_{x}^{(\alpha)} \cdots x_{0} I_{x}^{(\alpha)}}^{k \text { times }} f(x)
$$

for given condition $f(x) \in C_{\alpha}[a, b]$.

Local fractional Taylor expansion of the following functions is written as [32-36]

$$
E_{\alpha}\left(x^{\alpha}\right)=\sum_{k=0}^{\infty} \frac{x^{\alpha k}}{\Gamma(1+k \alpha)} .
$$

\section{The method}

In this section, we present the local fractional variational iteration method $[38,49,50]$ for handling differential equations with the help of the local fractional calculus theory [32-36].

Let us consider a general wave equation (1) subject to initial conditions as

$$
u(\zeta, 0)=\mu_{1}(\zeta), \quad L_{\xi}^{(\alpha)} u(\zeta, 0)=\mu_{2}(\zeta), \quad 0 \leq x \leq \zeta .
$$

We can construct a correction local fractional iteration algorithm given below

$$
u_{n+1}(\zeta, \xi)=u_{n}(\zeta, \xi)+{ }_{0} I_{\rho}^{(\alpha)} \frac{\lambda(\xi)^{\alpha}}{\Gamma(1+\alpha)}\left\{L_{\xi \xi}^{(2 \alpha)} u_{n}(\zeta, \xi)+R_{\xi}^{(\alpha)} u_{n}(\zeta, \xi)-g(\zeta, \xi)\right\}
$$

where $\lambda^{\alpha} / \Gamma(1+\alpha)$ is a general fractal Lagrange's multiplier.

By using the local fractional integration by parts [32], we obtain

$$
{ }_{0} I_{\rho}^{(\alpha)}\left\{\frac{\lambda(\xi)^{\alpha}}{\Gamma(1+\alpha)}\left[\frac{\partial^{\alpha} u_{n}(\zeta, \xi)}{\partial \xi^{\alpha}}\right]\right\}=\left.\frac{\lambda(\tau)^{\alpha}}{\Gamma(1+\alpha)} u_{n}(\zeta, \xi)\right|_{\xi=\rho}-{ }_{0} I_{\rho}^{(\alpha)}\left\{u_{n}(\zeta, \xi) \frac{\partial^{\alpha} \xi(\xi)}{\partial \xi^{\alpha}}\right\},
$$




$$
\begin{aligned}
& { }_{0} I_{\rho}^{(\alpha)}\left\{\frac{\lambda(\xi)^{\alpha}}{\Gamma(1+\alpha)}\left[\frac{\partial^{2 \alpha} u_{n}(\zeta, \xi)}{\partial \xi^{2 \alpha}}\right]\right\} \\
& =\left.\frac{\lambda(\tau)^{\alpha}}{\Gamma(1+\alpha)} \frac{\partial^{\alpha} u_{n}(\zeta, \xi)}{\partial \xi^{\alpha}}\right|_{\xi=\rho}-\left.u_{n}(\zeta, \xi) \frac{\partial^{\alpha}}{\partial \xi^{\alpha}} \frac{\lambda(\xi)^{\alpha}}{\Gamma(1+\alpha)}\right|_{\xi=\rho} \\
& \quad+{ }_{0} I_{t}^{(\alpha)}\left\{u_{n}(\zeta, \xi) \frac{\partial^{2 \alpha} \xi(\xi)}{\partial \xi^{2 \alpha}}\right\} .
\end{aligned}
$$

For the determination of the fractal Lagrange multiplier, the extremum condition of $u_{n+1}$ lead us to $\delta^{\alpha} u_{n+1}=0$. By making use of Eq. (25), we have

$$
\begin{aligned}
\delta^{\alpha} u_{n+1}(\zeta, \xi) & \\
= & \delta^{\alpha} u_{n}(\zeta, \xi)+\delta^{\alpha}{ }_{0} I_{\rho}^{(\alpha)} \frac{\lambda(\xi)^{\alpha}}{\Gamma(1+\alpha)}\left\{L_{\xi \xi}^{(2 \alpha)} u_{n}(\zeta, \xi)+R_{\xi}^{(\alpha)} u_{n}(\zeta, \xi)-g(\zeta, \xi)\right\} \\
= & \delta^{\alpha} u_{n}(\zeta, \xi)+\delta^{\alpha}{ }_{0} I_{\rho}^{(\alpha)} \frac{\lambda(\xi)^{\alpha}}{\Gamma(1+\alpha)}\left\{L_{\xi \xi}^{(2 \alpha)} u_{n}(\zeta, \xi)-L_{\xi}^{(\alpha)} u_{n}(\zeta, \xi)-g(\zeta, \xi)\right\} \\
= & \left(1-\left.\frac{\lambda(\xi)^{\alpha}}{\Gamma(1+\alpha)}\right|_{\xi=\rho}-\left.\frac{\partial^{\alpha}}{\partial \xi^{\alpha}} \frac{\lambda(\xi)^{\alpha}}{\Gamma(1+\alpha)}\right|_{\xi=\rho}\right) \delta^{\alpha} u_{n}(x, t)+\left.\frac{\lambda(\xi)^{\alpha}}{\Gamma(1+\alpha)}\right|_{\xi=\rho} \delta^{\alpha}\left\{\frac{\partial^{\alpha} u_{n}(\zeta, \xi)}{\partial \xi^{\alpha}}\right\} \\
& +{ }_{0} I_{\rho}^{(\alpha)}\left\{\delta^{\alpha} u_{n}(\zeta, \xi) \frac{\partial^{2 \alpha}}{\partial \xi^{2 \alpha}} \frac{\lambda(\xi)^{\alpha}}{\Gamma(1+\alpha)}\right\} .
\end{aligned}
$$

This yields to the stationary conditions listed below:

$$
\begin{aligned}
& 1-\left.\frac{\lambda(\xi)^{\alpha}}{\Gamma(1+\alpha)}\right|_{\xi=\rho}-\left.\frac{\partial^{\alpha}}{\partial \xi^{\alpha}} \frac{\lambda(\xi)^{\alpha}}{\Gamma(1+\alpha)}\right|_{\xi=\rho}=0, \\
& \left.\frac{\lambda(\xi)^{\alpha}}{\Gamma(1+\alpha)}\right|_{\xi=\rho}=0, \\
& \left.\frac{\partial^{2 \alpha}}{\partial \xi^{2 \alpha}} \frac{\lambda(\xi)^{\alpha}}{\Gamma(1+\alpha)}\right|_{\xi=\rho}=0 .
\end{aligned}
$$

Thus, we conclude that

$$
\frac{\lambda(\xi)^{\alpha}}{\Gamma(1+\alpha)}=\frac{(\xi-\rho)^{\alpha}}{\Gamma(1+\alpha)}
$$

From Eq. (28), the recurrence relation becomes

$$
u_{n+1}(\zeta, \xi)=u_{n}(\zeta, \xi)+{ }_{0} I_{\rho}^{(\alpha)} \frac{(\xi-\rho)^{\alpha}}{\Gamma(1+\alpha)}\left\{L_{\xi \xi}^{(2 \alpha)} u_{n}(\zeta, \xi)+R_{\xi}^{(\alpha)} u_{n}(\zeta, \xi)-g(\zeta, \xi)\right\}
$$

The function $u_{0}(\zeta, \xi)$ is selected by using the fractal initial conditions given as below:

$$
u_{0}(\zeta, \xi)=\mu_{1}(\zeta)+\frac{\xi^{\alpha}}{\Gamma(1+\alpha)} \mu_{2}(\zeta)
$$

Thus, the approximation expression becomes

$$
u(x, t)=\lim _{n \rightarrow \infty} \phi_{n}(x, t), \quad \lim _{n \rightarrow \infty} \phi_{n}(x, t)=\lim _{n \rightarrow \infty} \sum_{i=1}^{\infty} u_{i}(x, t) .
$$




\section{Solution of dissipative wave equation with a fractal string}

The dissipative wave equation with local fractional differential operator has the form

$$
\begin{aligned}
& L_{t t}^{(2 \alpha)} u(x, t)-L_{t}^{(\alpha)} u(x, t)-L_{x x}^{(2 \alpha)} u(x, t)-L_{x}^{(\alpha)} u(x, t)-\frac{t^{\alpha}}{\Gamma(1+\alpha)} \\
& \quad=0, \quad 0 \leq x \leq l, t>0
\end{aligned}
$$

subjected to the fractal initial conditions

$$
u(x, 0)=\frac{x^{2 \alpha}}{\Gamma(1+2 \alpha)}, \quad L_{t}^{(\alpha)} u(x, 0)=0, \quad 0 \leq x \leq l .
$$

Making use of Eq. (29), the recurrence relation reads as

$$
\begin{aligned}
u_{n+1}(x, t)= & u_{n}(x, t)+{ }_{0} I_{t}^{(\alpha)} \frac{(\tau-t)^{\alpha}}{\Gamma(1+\alpha)}\left\{L_{t t}^{(2 \alpha)} u_{n}(x, t)-L_{t}^{(\alpha)} u_{n}(x, t)\right\} \\
& -{ }_{0} I_{t}^{(\alpha)} \frac{(\tau-t)^{\alpha}}{\Gamma(1+\alpha)}\left\{L_{x x}^{(2 \alpha)} u_{n}(x, t)+L_{x}^{(\alpha)} u_{n}(x, t)+\frac{t^{\alpha}}{\Gamma(1+\alpha)}\right\} .
\end{aligned}
$$

If the expression from Eq. (30) is given, we can determine the fractal initial conditions, which are expressed through

$$
u_{0}(x, t)=u(x, 0)=\frac{x^{\alpha}}{\Gamma(1+\alpha)} .
$$

The first iteration yields

$$
\begin{aligned}
u_{1}(x, t)= & u_{0}(x, t)+{ }_{0} I_{t}^{(\alpha)} \frac{(\tau-t)^{\alpha}}{\Gamma(1+\alpha)}\left\{L_{t t}^{(2 \alpha)} u_{0}(x, t)-L_{t}^{(\alpha)} u_{0}(x, t)\right\} \\
& -{ }_{0} I_{t}^{(\alpha)} \frac{(\tau-t)^{\alpha}}{\Gamma(1+\alpha)}\left\{L_{x x}^{(2 \alpha)} u_{0}(x, t)+L_{x}^{(\alpha)} u_{0}(x, t)\right\}-{ }_{0} I_{t}^{(\alpha)} \frac{(\tau-t)^{\alpha}}{\Gamma(1+\alpha)} \frac{t^{\alpha}}{\Gamma(1+\alpha)} \\
= & \frac{x^{\alpha}}{\Gamma(1+\alpha)}+\frac{t^{2 \alpha}}{\Gamma(1+2 \alpha)}+\frac{t^{3 \alpha}}{\Gamma(1+3 \alpha)} .
\end{aligned}
$$

Thus, the second iteration reads

$$
\begin{aligned}
u_{2}(x, t)= & u_{1}(x, t)+{ }_{0} I_{t}^{(\alpha)} \frac{(\tau-t)^{\alpha}}{\Gamma(1+\alpha)}\left\{L_{t t}^{(2 \alpha)} u_{1}(x, t)-L_{t}^{(\alpha)} u_{1}(x, t)\right\} \\
& -{ }_{0} I_{t}^{(\alpha)} \frac{(\tau-t)^{\alpha}}{\Gamma(1+\alpha)}\left\{L_{x x}^{(2 \alpha)} u_{1}(x, t)+L_{x}^{(\alpha)} u_{1}(x, t)\right\}-{ }_{0} I_{t}^{(\alpha)} \frac{(\tau-t)^{\alpha}}{\Gamma(1+\alpha)} \frac{t^{\alpha}}{\Gamma(1+\alpha)} \\
= & \frac{x^{\alpha}}{\Gamma(1+\alpha)}+\left(\frac{t^{2 \alpha}}{\Gamma(1+2 \alpha)}+\frac{t^{3 \alpha}}{\Gamma(1+3 \alpha)}\right)+\left(\frac{t^{3 \alpha}}{\Gamma(1+3 \alpha)}+\frac{t^{4 \alpha}}{\Gamma(1+4 \alpha)}\right) .
\end{aligned}
$$

In similar manner, the third iteration is described by

$$
\begin{aligned}
u_{3}(x, t)= & u_{2}(x, t)+{ }_{0} I_{t}^{(\alpha)} \frac{(\tau-t)^{\alpha}}{\Gamma(1+\alpha)}\left\{L_{t t}^{(2 \alpha)} u_{2}(x, t)-L_{t}^{(\alpha)} u_{2}(x, t)\right\} \\
& -{ }_{0} I_{t}^{(\alpha)} \frac{(\tau-t)^{\alpha}}{\Gamma(1+\alpha)}\left\{L_{x x}^{(2 \alpha)} u_{2}(x, t)+L_{x}^{(\alpha)} u_{2}(x, t)\right\}-{ }_{0} I_{t}^{(\alpha)} \frac{(\tau-t)^{\alpha}}{\Gamma(1+\alpha)} \frac{t^{\alpha}}{\Gamma(1+\alpha)}
\end{aligned}
$$




$$
\begin{aligned}
= & \frac{x^{\alpha}}{\Gamma(1+\alpha)}+\left(\frac{t^{2 \alpha}}{\Gamma(1+2 \alpha)}+\frac{t^{3 \alpha}}{\Gamma(1+3 \alpha)}\right) \\
& +\left(\frac{t^{3 \alpha}}{\Gamma(1+3 \alpha)}+\frac{t^{4 \alpha}}{\Gamma(1+4 \alpha)}+\frac{t^{5 \alpha}}{\Gamma(1+5 \alpha)}\right) \\
= & \frac{x^{\alpha}}{\Gamma(1+\alpha)}+\left(\frac{t^{2 \alpha}}{\Gamma(1+2 \alpha)}+\frac{2 t^{3 \alpha}}{\Gamma(1+3 \alpha)}\right)+\frac{t^{4 \alpha}}{\Gamma(1+4 \alpha)}+\frac{t^{5 \alpha}}{\Gamma(1+5 \alpha)} .
\end{aligned}
$$

The fourth iteration is suggested by

$$
\begin{aligned}
u_{4}(x, t)= & u_{3}(x, t)+{ }_{0} I_{t}^{(\alpha)} \frac{(\tau-t)^{\alpha}}{\Gamma(1+\alpha)}\left\{L_{t t}^{(2 \alpha)} u_{3}(x, t)-L_{t}^{(\alpha)} u_{3}(x, t)\right\} \\
& -{ }_{0} I_{t}^{(\alpha)} \frac{(\tau-t)^{\alpha}}{\Gamma(1+\alpha)}\left\{L_{x x}^{(2 \alpha)} u_{3}(x, t)+L_{x}^{(\alpha)} u_{3}(x, t)\right\}-{ }_{0} I_{t}^{(\alpha)} \frac{(\tau-t)^{\alpha}}{\Gamma(1+\alpha)} \frac{t^{\alpha}}{\Gamma(1+\alpha)} \\
= & \frac{x^{\alpha}}{\Gamma(1+\alpha)}+\left(\frac{t^{2 \alpha}}{\Gamma(1+2 \alpha)}+\frac{t^{3 \alpha}}{\Gamma(1+3 \alpha)}+\frac{t^{4 \alpha}}{\Gamma(1+4 \alpha)}\right) \\
& +\left(\frac{t^{3 \alpha}}{\Gamma(1+3 \alpha)}+\frac{t^{4 \alpha}}{\Gamma(1+4 \alpha)}+\frac{t^{5 \alpha}}{\Gamma(1+5 \alpha)}+\frac{t^{6 \alpha}}{\Gamma(1+6 \alpha)}\right) \\
= & \frac{x^{\alpha}}{\Gamma(1+\alpha)}-\sum_{i=0}^{1} \frac{t^{i \alpha}}{\Gamma(1+i \alpha)}-\sum_{i=0}^{2} \frac{t^{i \alpha}}{\Gamma(1+i \alpha)} \\
& +\sum_{i=0}^{4} \frac{t^{i \alpha}}{\Gamma(1+i \alpha)}+\sum_{i=0}^{6} \frac{t^{i \alpha}}{\Gamma(1+i \alpha)} .
\end{aligned}
$$

The fifth approximation is written as follows:

$$
\begin{aligned}
u_{5}(x, t)= & u_{4}(x, t)+{ }_{0} I_{t}^{(\alpha)} \frac{(\tau-t)^{\alpha}}{\Gamma(1+\alpha)}\left\{L_{t t}^{(2 \alpha)} u_{4}(x, t)-L_{t}^{(\alpha)} u_{4}(x, t)\right\} \\
& -{ }_{0} I_{t}^{(\alpha)} \frac{(\tau-t)^{\alpha}}{\Gamma(1+\alpha)}\left\{L_{x x}^{(2 \alpha)} u_{4}(x, t)+L_{x}^{(\alpha)} u_{4}(x, t)\right\}-{ }_{0} I_{t}^{(\alpha)} \frac{(\tau-t)^{\alpha}}{\Gamma(1+\alpha)} \frac{t^{\alpha}}{\Gamma(1+\alpha)} \\
= & \frac{x^{\alpha}}{\Gamma(1+\alpha)}+\left(\frac{t^{2 \alpha}}{\Gamma(1+2 \alpha)}+\frac{t^{3 \alpha}}{\Gamma(1+3 \alpha)}+\frac{t^{4 \alpha}}{\Gamma(1+4 \alpha)}+\frac{t^{5 \alpha}}{\Gamma(1+5 \alpha)}\right) \\
& +\left(\frac{t^{3 \alpha}}{\Gamma(1+3 \alpha)}+\frac{t^{4 \alpha}}{\Gamma(1+4 \alpha)}+\frac{t^{5 \alpha}}{\Gamma(1+5 \alpha)}+\frac{t^{6 \alpha}}{\Gamma(1+6 \alpha)}+\frac{t^{7 \alpha}}{\Gamma(1+7 \alpha)}\right) \\
= & \frac{x^{\alpha}}{\Gamma(1+\alpha)}-\sum_{i=0}^{1} \frac{t^{i \alpha}}{\Gamma(1+i \alpha)}-\sum_{i=0}^{2} \frac{t^{i \alpha}}{\Gamma(1+i \alpha)} \\
& +\sum_{i=0}^{5} \frac{t^{i \alpha}}{\Gamma(1+i \alpha)}+\sum_{i=0}^{7} \frac{t^{i \alpha}}{\Gamma(1+i \alpha)} .
\end{aligned}
$$

Proceeding in this manner, we can derive the following formula:

$$
\begin{aligned}
u_{n}(x, t)= & u_{n-1}(x, t)+{ }_{0} I_{t}^{(\alpha)} \frac{(\tau-t)^{\alpha}}{\Gamma(1+\alpha)}\left\{L_{t t}^{(2 \alpha)} u_{n-1}(x, t)-L_{t}^{(\alpha)} u_{n-1}(x, t)\right\} \\
& -{ }_{0} I_{t}^{(\alpha)} \frac{(\tau-t)^{\alpha}}{\Gamma(1+\alpha)}\left\{L_{x x}^{(2 \alpha)} u_{n-1}(x, t)+L_{x}^{(\alpha)} u_{n-1}(x, t)\right\}-{ }_{0} I_{t}^{(\alpha)} \frac{(\tau-t)^{\alpha}}{\Gamma(1+\alpha)} \frac{t^{\alpha}}{\Gamma(1+\alpha)}
\end{aligned}
$$


Finally, the compact solution becomes

$$
u(x, t)=\frac{x^{\alpha}}{\Gamma(1+\alpha)}-\sum_{i=0}^{1} \frac{t^{i \alpha}}{\Gamma(1+i \alpha)}-\sum_{i=0}^{2} \frac{t^{i \alpha}}{\Gamma(1+i \alpha)}+2 E_{\alpha}\left(t^{\alpha}\right)
$$

\section{Solution of damped wave equation with a fractal string}

The damped wave equation with local fractional differential operator can be written in the form

$$
L_{t t}^{(2 \alpha)} u(x, t)-L_{t}^{(\alpha)} u(x, t)-L_{x x}^{(2 \alpha)} u(x, t)-\frac{x^{\alpha}}{\Gamma(1+\alpha)}=0, \quad 0 \leq x \leq l, t>0,
$$

and it is subjected to the initial conditions described by

$$
u(x, 0)=0, \quad L_{t}^{(\alpha)} u(x, 0)=-\frac{x^{\alpha}}{\Gamma(1+\alpha)}, \quad 0 \leq x \leq l .
$$

Applying Eq. (29), we arrive at the following iteration formula:

$$
\begin{aligned}
u_{n+1}(x, t)= & u_{n}(x, t)+{ }_{0} I_{t}^{(\alpha)} \frac{(\tau-t)^{\alpha}}{\Gamma(1+\alpha)}\left\{L_{t t}^{(2 \alpha)} u_{n}(x, t)-L_{t}^{(\alpha)} u_{n}(x, t)\right\} \\
& -{ }_{0} I_{t}^{(\alpha)} \frac{(\tau-t)^{\alpha}}{\Gamma(1+\alpha)}\left\{L_{x x}^{(2 \alpha)} u(x, t)+\frac{x^{\alpha}}{\Gamma(1+\alpha)}\right\} .
\end{aligned}
$$

By using Eq. (35), we obtain

$$
u_{0}(x, t)=-\frac{t^{\alpha}}{\Gamma(1+\alpha)} \frac{x^{\alpha}}{\Gamma(1+\alpha)} .
$$

Therefore, we deduce the first approximation as

$$
\begin{aligned}
u_{1}(x, t)= & u_{0}(x, t)+{ }_{0} I_{t}^{(\alpha)} \frac{(\tau-t)^{\alpha}}{\Gamma(1+\alpha)}\left\{L_{t t}^{(2 \alpha)} u_{0}(x, t)-L_{t}^{(\alpha)} u_{0}(x, t)\right\} \\
& -{ }_{0} I_{t}^{(\alpha)} \frac{(\tau-t)^{\alpha}}{\Gamma(1+\alpha)}\left\{L_{x x}^{(2 \alpha)} u_{0}(x, t)+\frac{x^{\alpha}}{\Gamma(1+\alpha)}\right\} \\
= & -\frac{t^{\alpha}}{\Gamma(1+\alpha)} \frac{x^{\alpha}}{\Gamma(1+\alpha)} .
\end{aligned}
$$

The second approximation has the form

$$
\begin{aligned}
u_{2}(x, t)= & u_{1}(x, t)+{ }_{0} I_{t}^{(\alpha)} \frac{(\tau-t)^{\alpha}}{\Gamma(1+\alpha)}\left\{L_{t t}^{(2 \alpha)} u_{1}(x, t)-L_{t}^{(\alpha)} u_{1}(x, t)\right\} \\
& -{ }_{0} I_{t}^{(\alpha)} \frac{(\tau-t)^{\alpha}}{\Gamma(1+\alpha)}\left\{L_{x x}^{(2 \alpha)} u_{1}(x, t)+\frac{x^{\alpha}}{\Gamma(1+\alpha)}\right\} \\
= & -\frac{t^{\alpha}}{\Gamma(1+\alpha)} \frac{x^{\alpha}}{\Gamma(1+\alpha)} .
\end{aligned}
$$


By using the same procedure, the third approximation becomes

$$
\begin{aligned}
u_{3}(x, t)= & u_{2}(x, t)++{ }_{0} I_{t}^{(\alpha)} \frac{(\tau-t)^{\alpha}}{\Gamma(1+\alpha)}\left\{L_{t t}^{(2 \alpha)} u_{2}(x, t)-L_{t}^{(\alpha)} u_{2}(x, t)\right\} \\
& -{ }_{0} I_{t}^{(\alpha)} \frac{(\tau-t)^{\alpha}}{\Gamma(1+\alpha)}\left\{L_{x x}^{(2 \alpha)} u_{2}(x, t)+\frac{x^{\alpha}}{\Gamma(1+\alpha)}\right\} \\
= & -\frac{t^{\alpha}}{\Gamma(1+\alpha)} \frac{x^{\alpha}}{\Gamma(1+\alpha)} .
\end{aligned}
$$

Thus, we have

$$
\begin{aligned}
& u_{0}(x, t)=-\frac{t^{\alpha}}{\Gamma(1+\alpha)} \frac{x^{\alpha}}{\Gamma(1+\alpha)}, \\
& u_{1}(x, t)=-\frac{t^{\alpha}}{\Gamma(1+\alpha)} \frac{x^{\alpha}}{\Gamma(1+\alpha)}, \\
& u_{0}(x, t)=-\frac{t^{\alpha}}{\Gamma(1+\alpha)} \frac{x^{\alpha}}{\Gamma(1+\alpha)}, \\
& \vdots \\
& u_{n}(x, t)=-\frac{t^{\alpha}}{\Gamma(1+\alpha)} \frac{x^{\alpha}}{\Gamma(1+\alpha)}
\end{aligned}
$$

and so on.

Finally, the solution is given by

$$
u(x, t)=-\frac{t^{\alpha}}{\Gamma(1+\alpha)} \frac{x^{\alpha}}{\Gamma(1+\alpha)} .
$$

\section{Conclusions}

In this manuscript, utilizing the local fractional differential operators, we investigated the damped and the dissipative wave equations in fractal strings. Based on the local fractional variational iteration method, the solutions of the damped and dissipative wave equations were presented. The iteration functions, which is local fractional continuous, is obtained easily within the fractal Lagrange multipliers, which can be optimally determined by the local fractional variational theory [32]. It is shown that the local fractional variational iteration method is an efficient and simple tool for handling partial differential equations with local fractional differential operator.

\section{Competing interests}

The authors declare that they have no competing interests.

\section{Authors' contributions}

Authors contributed equally and in writing this article. Authors read and approved the final manuscript.

\section{Author details}

${ }^{1}$ School of Mechanical Engineering, Tianjin University, Tianjin, 300072, China. ${ }^{2}$ Institute of Medical Equipment, Academy of Military Medical Sciences, Tianjin, 300161, China. ${ }^{3}$ Department of Mathematics and Computer Sciences, Faculty of Arts and Sciences, Cankaya University, Ankara, 06530, Turkey. ${ }^{4}$ Department of Chemical and Materials Engineering, Faculty of Engineering, King Abdulaziz University, P.O. Box 80204, Jeddah, 21589, Saudi Arabia. ${ }^{5}$ Institute of Space Sciences, Magurele, Bucharest, Romania. ${ }^{6}$ Department of Mathematics and Mechanics, China University of Mining and Technology, Xuzhou, Jiangsu 221008, China. ${ }^{7}$ Institute of Software Science, Zhengzhou Normal University, Zhengzhou, 450044, China. ${ }^{8}$ Institute of Applied mathematics, Qujing Normal University, Qujing, 655011, China. ${ }^{9}$ Department of Mathematics, Faculty of Mathematical Sciences, University of Mazandaran, Babolsar, Iran. 


\section{Acknowledgements}

Dedicated to Professor Hari M Srivastava.

The authors would like to thank the editor and the referees for their useful comments and remarks. The work is supported by the Natural Science Foundation of Tianjin, China (No. 10JCZDJC25100).

\section{Received: 23 January 2013 Accepted: 23 March 2013 Published: 10 April 2013}

\section{References}

1. He, JH: Variational iteration method - a kind of nonlinear analytical technique: some examples. Int. J. Non-Linear Mech. 34, 699-708 (1999)

2. He, JH: Some asymptotic methods for strongly nonlinear equations. Int. J. Mod. Phys. B 20, 1141-1199 (2006)

3. $\mathrm{He}, \mathrm{JH}, \mathrm{Wu}, \mathrm{XH}$ : Variational iteration method: new development and applications. Comput. Math. Appl. 54, 881-894 (2007)

4. He, $\mathrm{JH}$ : Approximate analytical solution for seepage flow with fractional derivatives in porous media. Comput. Methods Appl. Mech. Eng. 167(1-2), 57-68 (1998)

5. He, JH: Comment on 'Variational iteration method for fractional calculus using He's polynomials'. Abstr. Appl. Anal. 2012, 964974 (2012)

6. He, JH: Asymptotic methods for solitary solutions and compactons. Abstr. Appl. Anal. 2012, 916793 (2012)

7. Barari, A, Ghotbi, AR, Farrokhzad, F, Ganji, DD: Variational iteration method and homotopy-perturbation method for solving different types of wave equations. J. Appl. Sci. 8, 120-126 (2008)

8. Wazwaz, AM: The variational iteration method: a reliable analytic tool for solving linear and nonlinear wave equations. Comput. Math. Appl. 54, 926-932 (2007)

9. Momani, S, Abusaad, S: Application of He's variational-iteration method to Helmholtz equation. Chaos Solitons Fractals 27, 1119-1123 (2005)

10. Abdou, MA, Soliman, AA: Variational iteration method for solving Burgers' and coupled Burgers' equation. J. Comput. Appl. Math. 181, 245-251 (2005)

11. Abbasbandy, S: Numerical method for non-linear wave and diffusion equations by the variational iteration method. Int. J. Numer. Methods Eng. 73, 1836-1843 (2008)

12. Molliq, Y, Noorani, RMS, Hashim, MI: Variational iteration method for fractional heat-and wave-like equations. Nonlinear Anal., Real World Appl. 10, 1854-1869 (2009)

13. Hemeda, AA: Variational iteration method for solving wave equation. Comput. Math. Appl. 56, 1948-1953 (2008)

14. Batiha, B, Noorani, MSM, Hashim, I: Application of variational iteration method to heat-and wave-like equations. Phys. Lett. A 369, 55-61 (2007)

15. Biazar, J, Ghazvini, $\mathrm{H}$ : An analytical approximation to the solution of a wave equation by a variational iteration method. Appl. Math. Lett. 21, 780-785 (2008)

16. Wu, GC, Lee, EWM: Fractional variational iteration method and its application. Phys. Lett. A 374(25), 2506-2509 (2010)

17. He, JH: A short remark on fractional variational iteration method. Phys. Lett. A 375(38), 3362-3364 (2011)

18. $\mathrm{He}, \mathrm{JH}$ : Application of homotopy perturbation method to nonlinear wave equations. Chaos Solitons Fractals 26 695-700 (2005)

19. Jafari, H, Momani, S: Solving fractional diffusion and wave equations by modified homotopy perturbation method. Phys. Lett. A 370, 388-396 (2007)

20. He, JH, Wu, XH: Exp-function method for nonlinear wave equations. Chaos Solitons Fractals 30, 700-708 (2006)

21. Zhang, S: Application of Exp-function method to a KdV equation with variable coefficients. Phys. Lett. A 365, 448-453 (2007)

22. Odibat, ZM, Momani, S: Approximate solutions for boundary value problems of time-fractional wave equation. Appl. Math. Comput. 181, 767-774 (2006)

23. Datta, BK: A new approach to the wave equation - an application of the decomposition method. J. Math. Anal. Appl. 142, 6-12 (1989)

24. Momani, S: Analytical approximate solution for fractional heat-like and wave-like equations with variable coefficients using the decomposition method. Appl. Math. Comput. 165, 459-472 (2005)

25. Liao, SJ: Notes on the homotopy analysis method: some definitions and theorems. Commun. Nonlinear Sci. Numer. Simul. 4, 983-997 (2009)

26. Jafari, H, Seifi, S: Homotopy analysis method for solving linear and nonlinear fractional diffusion-wave equation. Commun. Nonlinear Sci. Numer. Simul. 14, 2006-2012 (2009)

27. Baleanu, D, Diethelm, K, Scalas, E, Trujillo, JJ: Fractional Calculus Models and Numerical Methods. Series on Complexity, Nonlinearity and Chaos. World Scientific, Boston (2012)

28. Ord, GN: Fractal space-time: a geometric analogue of relativistic quantum mechanics. J. Phys. A 16, 1869 (1999)

29. Marek-Crnjac, L: Polypseudologarithms and their applications to quantum ideal gas and the quantum wave collapse. Fractal Spacetime Noncommut. Geom. Quantum High Energy Phys. 2, $15-21$ (2012)

30. Hilfer, R: Exact solutions for a class of fractal time random walks. Fractals 3, 211-216 (1995)

31. Vrobel, S: Fractal time and fractal spacetime: phenomenology vs ontology. Fractal Spacetime Noncommut. Geom. Quantum High Energy Phys. 1, 41-44 (2011)

32. Yang, XJ: Advanced Local Fractional Calculus and Its Applications. World Science Publisher, New York (2012)

33. Yang, XJ: Local Fractional Functional Analysis and Its Applications. Asian Academic Publisher Limited, Hong Kong (2011)

34. Yang, XJ: Local fractional integral transforms. Prog. Nonlinear Sci. 4, 1-225 (2011)

35. Hu, MS, Agarwal, RP, Yang, XJ: Local fractional Fourier series with application to wave equation in fractal vibrating string. Abstr. Appl. Anal. 2012, 567401 (2012)

36. Yang, XJ, Baleanu, D, Zhong, WP: Approximation solution to diffusion equation on Cantor time-space. Proc. Rom. Acad., Ser. A (2013, in press)

37. Carpinteri, A, Sapora, A: Diffusion problems in fractal media defined on Cantor sets. Z. Angew. Math. Mech. 90 203-210(2010) 
38. Yang, XJ, Baleanu, D: Fractal heat conduction problem solved by local fractional variation iteration method. Therm. Sci. (2012). doi:10.2298/TSCI121124216Y

39. He, JH: A new fractal derivation. Therm. Sci. 15, 145-147 (2011)

40. Chen, W: Time-space fabric underlying anomalous diffusion. Chaos Solitons Fractals 28, 923-929 (2006)

41. Kolwankar, KM, Gangal, AD: Local fractional Fokker-Planck equation. Phys. Rev. Lett. 80, $214-217$ (1998)

42. Machado, JAT, Kiryakova, V, Mainardi, F: A poster about the recent history of fractional calculus. Fract. Calc. Appl. Anal. 13(3), 329-334 (2010)

43. Baleanu, D, Guvenç, ZB, Machado, JAT: New Trends in Nanotechnology and Fractional Calculus Applications. Springer Berlin (2009)

44. Baleanu, D, Machado, JAT, Luo, ACJ: Fractional Dynamics and Control. Springer, New York (2011)

45. Kilbas, AA, Srivastava, HM, Trujillo, JJ: Theory and Applications of Fractional Differential Equations. Elsevier, Amsterdam (2006)

46. Sabatier, J, Agrawal, OP, Machado, JAT: Advances in Fractional Calculus: Theoretical Developments and Applications in Physics and Engineering. Springer, New York (2007)

47. Podlubny, I: Fractional Differential Equations. Academic Press, New York (1999)

48. Mainardi, F: Fractional Calculus and Waves in Linear Viscoelasticity: An Introduction to Mathematical Models. Imperial College Press, London (2010)

49. Yang, XJ: Local fractional variational iteration method and its algorithms. Adv. Comput. Math. Appl. 1, 139-145 (2012)

50. Yang, YJ, Baleanu, D, Yang, XJ: A local fractional variational iteration method for Laplace equation within local fractional operators. Abstr. Appl. Anal. 2013, 202650 (2013)

51. Yang, XJ: The zero-mass renormalization group differential equations and limit cycles in non-smooth initial value problems. Prespacetime J. 3(9), 913-923 (2012)

52. Hu, MS, Baleanu, D, Yang, XJ: One-phase problems for discontinuous heat transfer in fractal media. Math. Probl. Eng. 2013, $358473(2013)$

doi:10.1186/1687-1812-2013-89

Cite this article as: Su et al.: Damped wave equation and dissipative wave equation in fractal strings within the local fractional variational iteration method. Fixed Point Theory and Applications 2013 2013:89.

\section{Submit your manuscript to a SpringerOpen ${ }^{\odot}$ journal and benefit from:}

- Convenient online submission

- Rigorous peer review

- Immediate publication on acceptance

Open access: articles freely available online

- High visibility within the field

- Retaining the copyright to your article 